\title{
Trading Strategies with Partial Access to the Derivatives Market
}

\begin{abstract}
This research analyzes implements derivative trading strategies when there are several assets and risk factors. We investigate portfolio improvements if investors have full and partial access to the derivatives markets, i.e. situations in which options are written on some but not all stocks traded in the market. The focus is on markets with jump risk. In these markets the choice of optimal exposure with respect to jump and diffusion risk is linked. In a numerical aplication we study the potential benefit from adding derivatives to the market. These considerations might be taken into account by exchanges planning to introduce new derivative contracts.
\end{abstract}

Key Words: Portfolio Choice, Jumps, Derivatives, Trading Strategies

JEL classification: G13 


\section{Introduction}

Optimal portfolio choice certainly belongs to one of the most extensively studied problems in finance. Merton $(1969,1971,1973)$ considers continuous time economies in which individuals dynamically adjust portfolio positions in order to maximize expected utility. More recent contributions addressing inter-temporal asset allocation include e.g. Cox and Huang (1989), Campbell (1993), Campbell and Viceira (1999), Daglesh (2002), Liu et al. (2003), Wu (2003), Munk and Sorensen (2004), Rudolf and Ziemba (2004), Chacko and Viceira (2005), Liu (2005).

Although portfolio selection is of major concern for many portfolio mangers it has taken a long time until there were attempts to include derivatives into the dynamic portfolio optimization ${ }^{1}$. This might partially be explained by the fact that initially, derivatives were seen as "redundant" securities which can be replicated by implementing a dynamic trading strategy in stocks and bonds. There are a number of derivatives pricing models including the celebrated Black/Scholes model supporting this view. In the recent literature additional risk factors such as stochastic volatility or jumps are taken into account. In these models, markets are incomplete and derivatives are no longer replicable by stocks and bonds alone. Instead they provide further information on risk premia and alow to speculate on new risk factors. This idea has first been addressed in a realistic model for a realistic set of derivatives by Liu and Pan (2003) and Branger et al. (2005) who develop models for a single stock economy and analyze implications of derivatives on portfolio management in the presence of stochastic jumps and volatility. Further examples for research in this direction include the papers of Bakshi and Madan (2000), Haugh and Lo (2001), and Carr et al. (2001).

Despite the existing work cited above, the analysis of derivatives in the context of portfolio selection is certainly at its very beginning. One immediate problem that needs to be investigated is the inclusion of several assets and derivatives. A manager responsible for the management of an investment fund is concerned about choosing optimal portfolio weights not of just one stock, one bond, and derivatives. Instead there are several investment opportunities (stocks, bonds, derivatives) which must be included in portfolio allocation. For instance, different stock markets may be driven by different risk factors: In January 2008, global stock markets were affected

\footnotetext{
${ }^{1}$ In static settings, the inclusion of derivatives has been analyzed e.g. by Ross (1976), Breeden and Litzenberger (1978), Arditti and John (1980), Green and Jarrow (1987).
} 
differently by the sub prime crises. Within 2 trading days the Eurostoxx 50 (Europe) and the Nikkei 225 (Japan) lost $6.1 \%$ and $9.3 \%$ respectively while the S\&P 500 (USA) decreased by $1.1 \%$ only $^{2}$. Furthermore, exchanges continuously try to develop new indices which address new risk factors. The German Exchange Deutsche Börse introduced e.g. the German Entrepreneurial Index GEX which is supposed to measure the market performance of companies led by entrepreneurs. One important purpose of these indices is to become underlyings of derivative securities which are traded on futures and options exchanges or which are issued as warrants by banks. Unfortunately, there are no considerations in the literature so far analyzing the potential need for derivatives on many different underlyings. The work of Liu and Pan (2003) and Branger et al. (2005) is limited in the sense that it only shows that derivatives can improve portfolio choice if they complete the market. However, they only distinguish between a market which is either complete or on which no derivatives are traded at all. They do not investigate the question of portfolio improvement as the market is gradually completed with derivatives on new underlyings.

The purpose of this paper is to set up a general model for portfolio selection when several stocks and derivatives are traded. We develop the notion of full and partial access to the derivatives market. In a situation of partial access the market is still incomplete due to a limited number of stocks which serve as underlyings for derivative securities. For both full and partial access to the derivatives market we determine asset allocation as the solution to a system of ordinary differential equations. We investigate the improvement of portfolio choice as additional derivatives become available for trade. The paper is structured as follows. Section 2 establishes a model for the case of full access to the derivatives market. Section 3 address the question of partial access in the presence of jumps. A numerical applications is discussed in section 4 . Finally, section 5 concludes.

\section{Full Access to the Derivatives Market}

In the economy a finite number $I$ of stocks are traded at prices $S \in \mathbb{R}^{I}$. The market is frictionless, i.e. there are no transaction costs, short selling is allowed, and stocks are perfectly divisible. Investors can both borrow and lend money at the risk-free interest rate $R$. Additionally, stochastic state variables $X$ on $\mathbb{R}^{L}$ are observed. More

\footnotetext{
${ }^{2}$ On Monday January 21, 2008, the markets in the US were closed due to a bank holiday. Compared to Friday January 18, 2008, Eurostoxx 50 and Nikkei 225 decreased by $7.3 \%$ and $3.9 \%$ respectively
} 
precisely, under the date generating measure $\mathcal{P}$ the market can be described by the following stochastic processes

$$
\begin{aligned}
d S_{i} & =S_{i}\left\{R+\sum_{j=1}^{J} a_{i, j} V_{j} \eta_{j}+\sum_{k=1}^{K} \mu_{i, k}\left[\nu^{\mathcal{P}} h_{k}^{\mathcal{P}}-\nu^{\mathcal{Q}} h_{k}^{\mathcal{Q}}\right]\right\} d t \\
& +S_{i} \sum_{j=1}^{J} a_{i, j} \sqrt{V_{j}} d W_{j}^{\mathcal{P}}+S_{i} \sum_{k=1}^{K} \mu_{i, k}\left(d N_{k}-\nu^{\mathcal{P}} h_{k}^{\mathcal{P}} d t\right) \\
d X_{l} & =m_{l}^{\mathcal{P}} d t+\sum_{j=1}^{J+L} b_{l, j} \sqrt{V_{j}} d W_{j}^{\mathcal{P}}+\sum_{k=1}^{K} \mu_{I+l, k} d N_{k} \\
V_{j} & =\bar{\beta}_{j}+\beta_{j} \cdot X \\
\nu^{\mathcal{P}} & =\bar{\lambda}^{\mathcal{P}}+\lambda^{\mathcal{P}} \cdot V \\
\nu^{\mathcal{Q}} & =\bar{\lambda}^{\mathcal{Q}}+\lambda^{\mathcal{Q}} \cdot V \\
R & =\bar{\rho}+\rho \cdot X \\
m_{l}^{\mathcal{P}} & =\bar{\varepsilon}_{l}^{\mathcal{P}}+\varepsilon_{l}^{\mathcal{P}} \cdot X
\end{aligned}
$$

where $V \in \mathbb{R}^{J+L}, \nu^{\mathcal{P}}, \nu^{\mathcal{Q}} \in \mathbb{R}$, and $m^{\mathcal{P}} \in \mathbb{R}^{L}$. As usual $V$ has the interpretation of a vector of stochastic factor variances. Furthermore, $a \in \mathbb{R}^{I \times J}, b \in \mathbb{R}^{L \times(J+L)}$, $\beta \in \mathbb{R}^{(J+L) \times L}, \rho \in \mathbb{R}^{L}, \varepsilon^{\mathcal{P}} \in \mathbb{R}^{L \times L}, \lambda^{\mathcal{P}}, \lambda^{\mathcal{Q}} \in \mathbb{R}^{J+L}, h^{\mathcal{P}}, h^{\mathcal{Q}} \in \mathbb{R}^{K}, \eta \in \mathbb{R}^{J+L}$, $\bar{\beta} \in \mathbb{R}^{J+L}, \bar{\varepsilon}^{\mathcal{P}} \in \mathbb{R}^{L}, \mu \in \mathbb{R}^{(I+L) \times K}$ as well as $\bar{\rho}, \bar{\lambda}^{\mathcal{P}}, \bar{\lambda}^{\mathcal{Q}} \in \mathbb{R}$ are deterministic, probably time dependent. Stochastic shocks are introduced by $W^{\mathcal{P}}$ which is a multidimensional standard Wiener process on $\mathbb{R}^{J+L}$ and the multivariate point process $N$ on $\mathbb{R}^{K}$. For some quantities the superscript $\mathcal{P}$ is introduced. This is to indicate that they might change when switching to a risk-neutral probability measure $\mathcal{Q}$ yet to be defined. The point process $N_{k}$ jumps with intensity $\nu^{\mathcal{P}} h_{k}$, i.e. the probability of a jump over a small time interval $\Delta t$ is approximately $\nu^{\mathcal{P}} h_{k} \Delta t$. If we require that $\sum_{k=1}^{K} h_{k}=1$ then $h_{k}$ is the probability that point process $N_{k}$ jumps conditional on a jump occurring. Thus, intuitively $\nu^{\mathcal{P}} \Delta t$ is approximately the probability that one of the point processes jumps during a short time interval $\Delta t$. Furthermore, $\nu^{\mathcal{Q}}$ and $h^{\mathcal{Q}}$ correspond to $\nu^{\mathcal{P}}$ and $h^{\mathcal{P}}$ under the risk neutral measure $\mathcal{Q}$. They will be discussed in greater detail below.

The stock prices are sensitive to the first $J$ diffusion factors. Furthermore, we observe $L$ state variables which are exposed to the additional risk factors. The state 
variables can e.g. be used to characterize the factor variance parameters $V_{j}, j \in$ $\{1, \ldots, J+L\}$, or the level of the short term interest rate $R$ which is applicable for borrowing and lending over the next time interval $d t$. Note that both $R$ and $V_{j}$ are linear functions in the state variables. The model nests several models with stochastic volatility and jumps as special cases like e.g. those of Merton (1976), Heston (1993), or Bakshi et al. (1997).

To ensure that the model (1) is well defined, we must prevent the stochastic factor variances $V_{j}$ to become negative. This condition puts some serious constraint on the model. A detailed discussion about possible model specifications is e.g. provided by Duffie and Kan (1996) who derive conditions to deal with the problem in pure diffusion settings. Throughout this paper we have to make the following assumption.

Assumption 1. The model is always properly defined such that the factor variances $V_{j}$ are always positive.

Examples for such models are presented in section 4. The market is per se incomplete with respect to the jump factors $N$ as well as to the diffusion factors $W_{J+1}^{\mathcal{P}}, \ldots, W_{J+L}^{\mathcal{P}}$. In such a situation the introduction of derivative securities might complete the market. Consider for instance the case of the Heston model discussed above. The stock price is sensitive to the first Wiener process $W_{1}$ but provides no exposure to $W_{2}^{\mathcal{P}}$. Only the variance $V_{1}=V_{2}=X_{1}$ are linked to $W_{2}^{\mathcal{P}}$. Since the price of a derivative security depends on the observed variance trading in such a contract enables the investor to generate arbitrary exposures to both diffusion factors. The market is complete and as a consequence any additional claim can now be replicated by trading in the stock, the bond, and the first derivative security. This example illustrates the reason for considering derivative securities in portfolio strategies in an incomplete market setting. In contrast to models which assume complete markets derivatives are no longer "redundant" securities which can be replicated by dynamic trading strategies in stocks and bonds only. Instead they provide the investor with the possibility to optimally chose exposures to all risk factors.

In incomplete markets the prices of derivatives are not unique. They depend on the pricing of diffusion and jump risk factors. In order to derive derivatives' prices and their stochastic processes we must specify the pricing kernel of the economy exogenously. 
Assumption 2. The pricing kernel $\pi$ of the economy follows the stochastic process

$$
\frac{d \pi}{\pi}=-R d t-\sum_{j=1}^{J+L} \sqrt{V_{j}} \eta_{j} d W_{j}^{\mathcal{P}}+\sum_{k=1}^{K}\left(\phi_{k}-1\right)\left(d N_{k}-\nu^{\mathcal{P}} h_{k}^{\mathcal{P}} d t\right)
$$

The specification of the pricing kernel is equivalent to selecting one of the infinitely many possible risk neutral measures under which each security is expected to earn the risk-free rate $R$ over the time interval $d t$. The vector $\phi \in \mathbb{R}^{K}$ characterizes the market price of jump risk. Under the new risk neutral measure $\mathcal{Q}$ the intensity parameters as well as the drift parameters for the state price process must be adjusted $^{3}$. More precisely the risk neutral intensity $\nu^{\mathcal{Q}}$ can be calculated as $\nu^{\mathcal{Q}}=\nu^{\mathcal{P}} h^{\mathcal{P}} \cdot \phi$. Thus, we can define $\lambda_{j}^{\mathcal{Q}}=\lambda_{j}^{\mathcal{P}} h^{\mathcal{P}} \cdot \phi$ and $\bar{\lambda}^{\mathcal{Q}}=\bar{\lambda}^{\mathcal{P}} h^{\mathcal{P}} \cdot \phi$ to set $\nu^{\mathcal{Q}}=\bar{\lambda}^{\mathcal{Q}}+\lambda^{\mathcal{Q}} \cdot V$. The risk neutral conditional probability $h_{k}^{\mathcal{Q}}$ that $N_{k}$ jumps given that a jump occurs follows then as $h_{k}^{\mathcal{Q}}=\frac{\nu^{\mathcal{P}} h_{k}^{\mathcal{P}} \phi_{k}}{\nu^{\mathcal{P}} h^{\mathcal{P}} \cdot \phi}=\frac{h_{k}^{\mathcal{P}} \phi_{k}}{h^{\mathcal{P}} \cdot \phi}$.

In the next step, we introduce the derivatives market. More precisely, we have to make an assumption concerning the properties of the derivative contracts traded. As discussed above, the existence of derivatives is connected to the question of market completeness.

Assumption 3. There are exactly $U=L+K$ derivatives available for trade which complete the market.

Note that after the introduction of the derivative securities any further financial contract that is introduced to the market becomes redundant and can be replicated by investing in stocks, bonds, and derivatives. There is a direct link between assumptions 2 and 3: If we observe the prices of the $L+K$ derivatives in a complete market we can immediately infer the market prices of risk and the pricing kernel. On the other hand if we know the pricing kernel we can directly price derivatives and derive their stochastic processes. Finally, we introduce different notions of access to the derivatives market.

Definition 1. Investors are said to have full access to the derivatives market if assumption 3 is fulfilled and their are no restrictions to buying and selling derivative securities.

\footnotetext{
${ }^{3}$ See e.g. Björk et al. (1997), Theorem 3.12 for further details.
} 
We consider an investor with an initial positive wealth $\Pi(0)$ and full access to the derivatives market. At each time $t$ the investor chooses to invest a fraction of $\omega_{i}(t)$ into the $i$ th stock and $\psi_{u}(t)$ into the $u$ th derivative, $\omega \in \mathbb{R}^{I}, \psi \in \mathbb{R}^{U}$. The investor has planning horizon $T$. It is the objective to maximize expected utility of the terminal wealth at time $T$

$$
\max _{\omega(t), \psi(t), 0 \leq t \leq T} \mathbb{E}\left[\frac{\Pi(T)^{1-\gamma}}{1-\gamma}\right] .
$$

The coefficient $\gamma>0$ is the coefficient of the investor's relative risk aversion. This is a variation of a dynamic portfolio planning problem which was originally analyzed by Merton (1971).

Lemma 1. Let $O_{u}(t)$ be the price of the uth derivative available for trade. Imposing self-financing condition the stochastic process for the wealth is given by

$$
\frac{d \Pi}{\Pi}=R d t+\sum_{j=1}^{J+L} \theta_{j}^{(W)}\left(\sqrt{V_{j}} d W_{j}^{\mathcal{P}}+\eta_{j} V_{j} d t\right)+\sum_{k=1}^{K} \theta_{k}^{(N)} d Z_{k}
$$

where

$$
\begin{aligned}
& \theta_{j}^{(W)}=\left\{\begin{array}{l}
\sum_{u=1}^{U} \psi_{u} \frac{1}{O_{u}}\left(\sum_{i=1}^{I} \frac{\partial O_{u}}{\partial S_{i}} a_{i, j} S_{i}+\sum_{l=1}^{L} \frac{\partial O_{u}}{\partial X_{l}} b_{l, j}\right) \\
+\sum_{i=1}^{I} \omega_{i} a_{i, j}, \quad \text { if } j \in\{1, \ldots, I\} \\
\sum_{u=1}^{U} \psi_{u} \frac{1}{O_{u}} \sum_{l=1}^{L} \frac{\partial O_{u}}{\partial X_{l}} b_{l, j}, \quad \text { if } j \in\{J+1, \ldots, J+L\}
\end{array}\right. \\
& \theta_{k}^{(N)}=\sum_{i=1}^{I} \omega_{i} \mu_{i, k}+\sum_{u=1}^{U} \psi_{u} \frac{1}{O_{u}} \Delta_{k} O_{u} .
\end{aligned}
$$

Furthermore, $d Z_{k}$ is defined as $d Z_{k}=d N_{k}-\nu^{\mathcal{P}} h_{k}^{\mathcal{P}} d t+\left(\nu^{\mathcal{P}} h_{k}^{\mathcal{P}}-\nu^{\mathcal{Q}} h_{k}^{\mathcal{Q}}\right) d t$ and $\Delta_{k} O_{u}=O_{u}\left(S_{1}\left(1+\mu_{1, k}\right), \ldots, X_{L}\left(1+\mu_{I+L, k}\right)\right)-O_{u}\left(S_{1}, \ldots, X_{L}\right)$.

Proof: See Appendix.

Intuitively, the $\theta^{(\cdot)}$ can be seen as the total portfolio exposures to particular risk factors. If investors have full access to the derivatives market then their is a one to one relationship between the portfolio weights $\omega$ and $\psi$ on the one hand and $\theta^{(W)}$ as well as $\theta^{(N)}$ on the other. To put it differently, if we know the portfolio weights we can of course compute the associated factor exposures. Turning the argument around, if we are targeting a particular factor exposure then we can solve for the corresponding portfolio weights. This is a particularly useful result because then we 
can optimize the factor exposures and calculate the optimal portfolio weights by determining (5) $\theta^{(W)}$ and $\theta^{(N)}$ instead of $\omega$ and $\psi$.

Considering factor exposures (4) implies a constraint on portfolio choice. Note that wealth may become negative if there is a $k$ such that $\theta_{k}^{(N)}<-1$. This is a particular problem faced by investors in the context of jump-diffusion models. In contrast to pure diffusion models in which investors can adjust portfolios quickly enough to avoid ruin, negative wealth is possible if jumps are large enough. If one of the jump factor exposures is smaller than -1 the investor faces the risk of losing more than his or her total wealth instantaneously without having the possibility to react quickly enough by shifting portfolio weights. Therefore, we introduce the following definition.

Definition 2. A portfolio strategy is admissible if $\forall k: \theta_{k}^{(N)}>-1$.

Having stated the definition for admissible portfolio strategies for ease of exposition we make the following assumption.

Assumption 4. The optimal portfolio strategy is admissible.

This assumption ensures that the investor does not face the risk that wealth may jump negative. It is necessary in order to enable an unconstraint optimization of the factor risk exposures in the following. This facilitates our analysis. We will return to this assumption shortly and show that it always holds in the optimum.

In order to solve the portfolio choice problem we proceed according to Merton (1971). We define the indirect utility function as

$$
\mathcal{J}(t, \Pi, X)=\max _{\theta^{(\cdot)}} \mathbb{E}\left(\frac{\Pi^{1-\gamma}(T)}{1-\gamma} \mid \Pi=\Pi(t), X=X(t)\right) .
$$

As described earlier we maximize over the factor exposures. The corresponding portfolio weights follow immediately from Lemma 1: A system of linear equations must be solved such that we "extract" the portfolio weights from the risk factor exposures.

The solution to the optimization problem is summarized in the following proposition. It is obtained by conjecturing a functional form for the indirect utility function. The applicability of this function can be proofed by showing that it fulfils the relevant Hamilton/Jacobi/Bellman equation. 
Proposition 1. Let the stock price process and the pricing kernel be given by (1) and (2) respectively. Then the indirect utility function is given by

$$
\mathcal{J}(t, \Pi, X)=\frac{\Pi^{1-\gamma}}{1-\gamma} e^{\gamma \bar{H}(t)+\gamma H(t) \cdot X}
$$

where the functions $\bar{H}(t)$ and $H_{z}(t), z \in\{1, \ldots, L\}$ fulfil the following system of ordinary differential equations

$$
\begin{aligned}
\frac{\partial \bar{H}}{\partial t} & =-\frac{1}{2} H^{T} \Psi_{0} H-\Gamma_{0} \cdot H+\zeta_{0} \\
\frac{\partial H_{z}}{\partial t} & =-\frac{1}{2} H^{T} \Psi_{z} H-\Gamma_{z} \cdot H+\zeta_{z}
\end{aligned}
$$

subject to the boundary conditions $\bar{H}(T)=H_{z}(T)=0$ with

$$
\begin{aligned}
\zeta_{0} & =-\frac{1-\gamma}{\gamma}\left[\bar{\rho}+\frac{1}{2} \sum_{j=1}^{J+L} \frac{\eta_{j}^{2}}{\gamma} \bar{\beta}_{j}\right] \\
& +\frac{1-\gamma}{\gamma}\left(\bar{\lambda}^{\mathcal{Q}}+\sum_{j=1}^{J} \lambda_{j}^{\mathcal{Q}} \bar{\beta}_{j}\right) \sum_{k=1}^{K}\left[\left(\frac{1}{\phi_{k}}\right)^{\frac{1}{\gamma}} e^{\sum_{u=1}^{L} H_{u} \mu_{I+u, k}}-1\right] h_{k}^{\mathcal{Q}} \\
& -\frac{\left(\bar{\lambda}^{\mathcal{P}}+\sum_{j=1}^{J} \lambda_{j}^{\mathcal{P}} \bar{\beta}_{j}\right)}{\gamma} \sum_{k=1}^{K}\left[\left(\frac{1}{\phi_{k}}\right)^{\frac{1}{\gamma}} \phi_{k} e^{\sum_{u=1}^{L} H_{u} \mu_{I+u, k}}-1\right] h_{k}^{\mathcal{P}}
\end{aligned}
$$




$$
\begin{gathered}
\zeta_{z}=-\frac{1-\gamma}{\gamma}\left[\rho_{z}+\frac{1}{2} \sum_{j=1}^{J+L} \frac{\eta_{j}^{2}}{\gamma} \beta_{j, z}\right] \\
+\frac{1-\gamma}{\gamma} \sum_{k=1}^{K}\left[\left(\frac{1}{\phi_{k}}\right)^{\frac{1}{\gamma}} e^{\sum_{u=1}^{L} H_{u} \mu_{I+u, k}}-1\right] \sum_{j=1}^{J+L} \lambda_{j}^{\mathcal{Q}} \beta_{j, z} h_{k}^{\mathcal{Q}} \\
-\frac{1}{\gamma} \sum_{k=1}^{K}\left[\left(\frac{1}{\phi_{k}}\right)^{\frac{1}{\gamma}} \phi_{k} e^{\sum_{u=1}^{L} H_{u} \mu_{I+u, k}}-1\right] \sum_{j=1}^{J+L} \lambda_{j}^{\mathcal{P}} \beta_{j, z} h_{k}^{\mathcal{P}} \\
\left(\Gamma_{0}\right)_{l}=\bar{\varepsilon}_{l}^{\mathcal{P}}+\frac{1-\gamma}{\gamma} \sum_{j=1}^{J+L} \eta_{j} b_{l, j} \bar{\beta}_{j} \\
\left(\Gamma_{z}\right)_{l}=\varepsilon_{l, z}^{\mathcal{P}}+\frac{1-\gamma}{\gamma} \sum_{j=1}^{J+L} \eta_{j} b_{l, j} \beta_{j, z} \\
\left(\Psi_{0}\right)_{i, j}=\sum_{k=1}^{J+L} \bar{\beta}_{k} b_{i, k} b_{j, k} \\
\left(\Psi_{z}\right)_{i, j}=\sum_{k=1}^{J+L} \beta_{k, z} b_{i, k} b_{j, k}
\end{gathered}
$$

Note that $\Psi_{0}, \Psi_{z} \in \mathbb{R}^{L \times L}, \Gamma_{0}, \Gamma_{z} \in \mathbb{R}^{L}$, and $\zeta_{0}, \zeta_{z} \in \mathbb{R}$.

The optimal factor exposures are

$$
\begin{aligned}
\theta_{j}^{(W) *} & =\frac{\eta_{j}}{\gamma}+\sum_{l=1}^{L} H_{l} b_{l, j} \\
\theta_{k}^{(N) *} & =\left(\frac{1}{\phi_{k}}\right)^{\frac{1}{\gamma}} e^{\sum_{u=1}^{L} H_{u} \mu_{J+u, k}}-1
\end{aligned}
$$

Proof: See Appendix.

The system of ordinary differential equations (7) is of the Ricatti type and as such solvable. For some special cases solutions are available in closed-form. Others can be solved efficiently by applying numerical techniques such as a Runge-Kutta method. The trading strategies implied are always admissible if $\forall k: \phi_{k}>0$ which must be the case because otherwise jump-intensities for some jump processes may become negative under the risk neutral measure. Assumption 4 is fulfilled and the 
trading strategies identified are indeed admissible. The single stock models of Liu and Pan (2003) and Branger et al. (2005) can be seen as special cases of our model. Thus their solutions nest in proposition 1.

Equation (8) contains the optimal factor exposures. Optimal portfolio weights are linked to these optimal factor exposures through lemma 1 and depend on the characteristics of the particular derivative securities which complete the market. As stated above, once optimal factor exposures and exact contract specifications of the derivatives are known the determination of optimal portfolio weights is rather straightforward and can be carried out by solving a set of linear equations.

\section{Partial Access to the Derivatives Market}

In real world markets only a limited number of derivative securities is available. In this case investors might only select their ideal exposures to some but not all risk factors due to the fact that the market is still incomplete. We adopt the notion of partial access to the derivatives' market which is made precise in the next definition.

Definition 3. Investors are said to have partial access to the derivatives market if assumption 3 does not hold, i.e. after the introduction of derivatives the market is still incomplete.

In the situation of partial access the market may be incomplete with respect to an extra diffusion and/or jump factor.

In the following, we restrict ourselves to the analysis of a jump diffusion model for stock prices without any further state variables which could introduce stochastic volatilities, interest rates and the like. This is only for ease of exposition and in order to keep results as simple as possible. Further stochastic variables could be introduced without major difficulties.

Without loss of generality we set $V_{j}=1, \lambda_{j}^{(\cdot)}=0, \forall j \in\{1, \ldots, J\}$. The stochastic 
process for the stock prices (1) reduces to

$$
\begin{aligned}
d S_{i} & =S_{i}\left\{R+\sum_{j=1}^{J} a_{i, j} \eta_{j}+\sum_{k=1}^{K} \mu_{i, k}\left[\bar{\lambda}^{\mathcal{P}} h_{k}^{\mathcal{P}}-\bar{\lambda}^{\mathcal{Q}} h_{k}^{\mathcal{Q}}\right]\right\} d t \\
& +S_{i} \sum_{j=1}^{J} a_{i, j} d W_{j}^{\mathcal{P}}+S_{i} \sum_{k=1}^{K} \mu_{i, k}\left(d N_{k}-\bar{\lambda}^{\mathcal{P}} h_{k}^{\mathcal{P}} d t\right) .
\end{aligned}
$$

We assume that investors have only partial access to the derivatives market i.e. the market is complete only with respect to the jump factors $k \in \mathbb{K} \subset\{1, \ldots, K\}$. Liu et al. (2003) analyze the case of a single stock market with jump risk. In their model the investor is faced with the investment problem of maximizing the joint exposure to diffusion and jump risk. If derivatives are introduced they can be used to disentangle these types of risk factors from each other. In a market with full access the jump and diffusion risks can be disentangled completely, i.e. each factor exposure to jump and diffusion risk can be selected optimally according to the risk preferences. The picture changes in the case of partial access to the derivatives market. This is made precise in the following assumption replacing assumption 3 in this paragraph

Assumption 5. There is partial access to the derivatives markets. Only the factor exposures to jump and diffusion risk $\theta_{k}^{(N)}, k \in \mathbb{K}$ and $\theta_{j}^{(W)}, j \in \mathbb{M} \subset\{1, \ldots, J\}$ can be chosen independently of each other.

The jump and diffusion risk factor exposures which cannot be chosen freely are linked directly to each other, i.e. a choice in the risk factor exposures implies a certain choice in the remaining diffusion risk exposures and vice versa. As such the diffusion risk factor exposures which cannot be freely chosen become functions of the jump risk exposures.

In order to solve the portfolio optimization problem we consider again the functional form of the indirect utility function defined in (5). In the situation discussed here the investor maximizes over all jump factor exposures and the free diffusion factor exposures, i.e.

$$
\mathcal{J}(t, \Pi)=\max _{\theta_{k}^{(N)}, \theta_{j}^{(W)}, j \in \mathbb{M}} \mathbb{E}\left(\frac{\Pi^{1-\gamma}(T)}{1-\gamma} \mid \Pi=\Pi(t)\right)
$$

subject to the constraint that the trading strategy is admissible. 
Proposition 2. If there is only partial access to the derivatives market, assumption 5 holds and there are no additional state variables other than the stock prices, and $V_{j}=1, \lambda_{j}^{(\cdot)}=0, \forall j \in\{1, \ldots, J\}$ then the indirect utility function is given by

$$
\mathcal{J}(t, \Pi)=\frac{\Pi^{1-\gamma}}{1-\gamma} e^{\gamma \bar{H}(t)}
$$

with

$$
\begin{aligned}
\frac{\partial H(t)}{\partial t} & =-\frac{1-\gamma}{\gamma}\left[R+\sum_{j=1}^{J} \theta_{j}^{(W) *} \eta_{j}-\bar{\lambda}^{\mathcal{Q}} \sum_{k=1}^{K} \theta_{k}^{(N) *} h_{k}^{\mathcal{Q}}\right] \\
& +\frac{1}{2}(1-\gamma)\left(\theta_{k}^{(W) *}\right)-\bar{\lambda}^{\mathcal{P}} \sum_{k=1}^{K} \frac{\left(1+\theta_{k}^{(N) *}-1\right)}{\gamma} h_{k}^{\mathcal{P}}
\end{aligned}
$$

the optimal factor exposures for all $j \in \mathbb{M}$ and for all $k \in \mathbb{K}$ are given by

$$
\begin{aligned}
\theta_{j}^{(W) *} & =\frac{\eta_{j}}{\gamma} \\
\theta_{k}^{(N) *} & =\left(\frac{1}{\phi_{k}}\right)^{\frac{1}{\gamma}}-1
\end{aligned}
$$

The exposures for the other jump factors are chosen such that it holds

$$
\bar{\lambda}^{\mathcal{P}} h_{k}^{\mathcal{P}}\left(1+\theta_{k}^{(N) *}\right)^{-\gamma}=\sum_{j \notin \mathbb{M}} \frac{\partial \theta_{j}^{(W) *}}{\partial \theta_{k}^{(N) *}}\left(\theta_{j}^{(W) *} \gamma-\eta_{j}\right)+\bar{\lambda}^{\mathcal{Q}} h_{k}^{\mathcal{Q}}
$$

for all $k \notin \mathbb{K}$, where $\sum_{j \notin \mathbb{M}}$ is the sum over all $j$ not in $\mathbb{M}$ subject to the constraint that the trading strategy is admissible. The exposures $\theta_{j}^{(W)}, j \notin \mathbb{M}$ follow as functions of the jump risk exposures.

Proof. See Appendix.

If all model parameters are constant the solution to the ordinary differential equations (12) is straightforward and $H(t)$ can be determined in closed-form once the optimal risk factor exposures are known. An example is shown below. Note that $H(t)$ is state independent. This is due to the fact that we have excluded any further state variables from the analysis. Liu et al. (2003) analyze a single stock situation 
with jumps and stochastic volatility. In their model the jump intensity is a linear function of the stochastic variance which leads to state dependency of the optimal portfolio weights. For ease of exposition related issues were not taken into account here. However, they can be incorporated into the model without major difficulties. Proposition 2 extends the work of Liu et al. (2003) in the sense that if there is no access to the derivatives market optimal portfolio weights for the several asset case can be determined ${ }^{4}$. Their original model considers just one asset which is available for trade.

\section{Numerical Application}

In this section we present applications and comparative statics analysis for the model described in the previous sections. In our example we investigate a situation in which two stocks are traded. These stocks could represent different stock markets, a general and a (new) specialized stock portfolio on which derivatives are to be introduced, different industries, etc. In a broader sense they could also be seen as different asset classes or investment opportunities like equity, commodities, or foreign exchange.

In order to measure portfolio improvement we have to select a performance measure. We follow Liu and Pan (2003) and define improvement in terms of the annualized, continuously compounded return in certainty equivalent wealth. The certainty equivalent wealth is set as

$$
\Pi^{C E W}=\Pi(0) e^{\frac{\gamma}{1-\gamma}(\bar{H}+H \cdot X)}
$$

A situation with full market access is given if there are derivatives for trade on both stocks. We may have partial access to the derivatives market if there are only derivatives on one stock. Finally, there is no access to the derivatives market if there are no derivatives traded. In this sense three different certainty equivalent wealths can be determined including certainty equivalent wealth with full $\Pi_{\text {full }}^{C E W}$, partial $\Pi_{\text {partial }}^{C E W}$, and no access $\Pi_{\text {no }}^{C E W}$. Therefore, we have two improvement measures, one for full access to the derivatives market and one for partial access. They are given

${ }^{4}$ E.g. their example "A. Constant Volatility and Deterministic Jump Size" can be seen as special case of Proposition 2 when there is one asset and no derivative traded. 
by

$$
\begin{aligned}
\mathcal{R}_{\mathrm{full}} & =\frac{\ln \prod_{\mathrm{full}}^{C E W}-\ln \prod_{\mathrm{no}}^{C E W}}{\tau} \\
\mathcal{R}_{\text {partial }} & =\frac{\ln \prod_{\mathrm{partial}}^{C E W}-\ln \prod_{\mathrm{no}}^{C E W}}{\tau},
\end{aligned}
$$

where $\tau=T-t$. To characterize the contribution through the introduction of each derivative we set

$$
\begin{aligned}
\text { Derivative }_{1} & =\mathcal{R}_{\text {partial }} \\
\text { Derivative }_{2} & =\mathcal{R}_{\text {full }}-\mathcal{R}_{\text {partial }}
\end{aligned}
$$

Derivative $_{1}$ is identical with $\mathcal{R}_{\text {partial }}$ because the total improvement is due to the first derivative introduced in the case of partial access. Derivative ${ }_{2}$ measures the extra-improvement by introducing a second derivative. It is given by the difference of $\mathcal{R}_{\text {partial }}$ and $\mathcal{R}_{\text {full }}$.

For simplicity we assume that interest rates and instantaneous factor variances are constant, i.e. there are no state variables other than stock prices. Jump amplitudes are fixed at $\mu_{1}$ and $\mu_{2}$ for stock prices $S_{1}$ and $S_{2}$ respectively. Furthermore, jump intensities under the empirical and the risk neutral measure are constant. In total, there are two jump processes driving stock price movements $(K=2)$. More precisely, market dynamics can be described by the following stochastic stock price processes

$$
\begin{aligned}
d S_{1} & =S_{1}\left\{r+a_{1,1} \eta_{1}+\left(\bar{\lambda}^{\mathcal{P}} h_{1}^{\mathcal{P}}-\bar{\lambda}^{\mathcal{Q}} h_{1}^{\mathcal{Q}}\right) \mu_{1}\right\} d t+S_{1} a_{1,1} d W_{1}^{\mathcal{P}} \\
& +S_{1} \mu_{1}\left(d N_{1}-\bar{\lambda}^{\mathcal{P}} h_{1}^{\mathcal{P}} d t\right) \\
d S_{2} & =S_{2}\left\{r+a_{2,1} \eta_{1}+a_{2,2} \eta_{2}+\left(\bar{\lambda}^{\mathcal{P}}-\bar{\lambda}^{\mathcal{Q}}\right) \mu_{2}\right\} d t+S_{2} a_{2,1} d W_{1}^{\mathcal{P}} \\
& +S_{2} a_{2,2} d W_{2}^{\mathcal{P}}+S_{2} \mu_{2}\left[\left(d N_{1}-\bar{\lambda}^{\mathcal{P}} h_{1}^{\mathcal{P}} d t\right)+\left(d N_{2}-\bar{\lambda}^{\mathcal{P}} h_{2}^{\mathcal{P}} d t\right)\right] .
\end{aligned}
$$

This means that the conditional probability that a jump is a common jump of both stocks is $h_{1}^{(\mathcal{P})}$ (empirical measure) or $h_{1}^{(\mathcal{Q})}$ (risk neutral measure). If there are two derivatives - one on stock 1 and one on stock 2 - then the market is complete and all factor exposures can be chosen independently of each other. For instance, let 
$O_{1}$ and $O_{2}$ be the prices of call options written on stock 1 and 2 respectively ${ }^{5}$. Per Lemma 1, factor exposures are

$$
\begin{aligned}
\theta_{1}^{(W)} & =\psi_{1} \frac{1}{O_{1}} \frac{\partial O_{1}}{\partial S_{1}}+\omega_{1} a_{1,1}+\psi_{2} \frac{1}{O_{2}} \frac{\partial O_{2}}{\partial S_{2}} a_{2,1} S_{2}+\omega_{2} a_{2,1} \\
\theta_{2}^{(W)} & =\psi_{2} \frac{1}{O_{2}} \frac{\partial O_{2}}{\partial S_{2}} a_{2,2} S_{2}+\omega_{2} a_{2,2} \\
\theta_{1}^{(N)} & =\psi_{1} \frac{1}{O_{1}} \Delta O_{1}+\omega_{1} \mu_{1}+\psi_{2} \frac{1}{O_{2}} \Delta O_{2}+\omega_{2} \mu_{2} \\
\theta_{2}^{(N)} & =\psi_{2} \frac{1}{O_{2}} \Delta O_{2}+\omega_{2} \mu_{2}
\end{aligned}
$$

with $\Delta O_{1}=\Delta_{1} O_{1}$ and $\Delta O_{2}=\Delta_{1} O_{2}=\Delta_{2} O_{2}$. Now, we can optimize $\theta_{1}^{(W)}, \theta_{2}^{(W)}, \theta_{1}^{(N)}, \theta_{2}^{(N)}$ and adjust parameters $\omega_{1}, \omega_{2}, \psi_{1}, \psi_{2}$ such that the portfolio exhibits these factors weights.

If there are no derivatives on stock 2 then the situation changes. This time factor exposures of the portfolio are

$$
\begin{aligned}
\theta_{1}^{(W)} & =\psi_{1} \frac{1}{O_{1}} \frac{\partial O_{1}}{\partial S_{1}}+\omega_{1} a_{1,1}+\omega_{2} a_{2,1} \\
\theta_{2}^{(W)} & =\omega_{2} a_{2,2} \\
\theta_{1}^{(N)} & =\psi_{1} \frac{1}{O_{1}} \Delta O_{1}+\omega_{1} \mu_{1}+\omega_{2} \mu_{2} \\
\theta_{2}^{(N)} & =\omega_{2} \mu_{2} .
\end{aligned}
$$

That means that by fixing $\omega_{2}$ we select both $\theta_{2}^{(W)}$ and $\theta_{2}^{(N)}$. Put it the other way around if we pick $\omega_{2}$ in order to match the diffusion factor exposure $\theta_{2}^{(W)}$ then at the same time we make a choice for $\theta_{2}^{(N)}$. In other words, jump and diffusion factors are no longer disentangled. Instead, $\theta_{2}^{(W)}$ becomes a function of $\theta_{2}^{(N)}$ (or the opposite way). Nevertheless, due the derivative on stock 1 we still can select $\theta_{1}^{(W)}$ and $\theta_{1}^{(N)}$ independently of each other.

\footnotetext{
${ }^{5}$ It is not essential that the derivatives considered are call options. In fact, they could be arbitrary contracts with $\frac{\partial O_{1}}{\partial S_{1}} \neq 0, \frac{\partial O_{2}}{\partial S_{2}} \neq 0, \Delta_{1} O_{1} \neq 0, \Delta_{1} O_{2}=\Delta_{2} O_{2} \neq 0$, and $\frac{\partial O_{1}}{\partial S_{2}}=\frac{\partial O_{2}}{\partial S_{1}}=\Delta_{2} O_{1}=0$.
} 
Finally, if there is no access to the derivatives market, factor exposures become

$$
\begin{aligned}
& \theta_{1}^{(W)}=\omega_{1} a_{1,1}+\omega_{2} a_{2,1} \\
& \theta_{2}^{(W)}=\omega_{2} a_{2,2} \\
& \theta_{1}^{(N)}=\omega_{1} \mu_{1}+\omega_{2} \mu_{2} \\
& \theta_{2}^{(N)}=\omega_{2} \mu_{2} .
\end{aligned}
$$

Again $\theta_{2}^{(W)}$ and $\theta_{2}^{(N)}$ are directly related to each other through $\omega_{2}$. The same is true for $\theta_{1}^{(W)}$ and $\theta_{1}^{(N)}$ which are both influenced by $\omega_{1}$. Furthermore, $\omega_{2}$ also affects the factor exposures $\theta_{1}^{(W)}$ and $\theta_{1}^{(N)}$ turning portfolio choice into an even more complicated matter. Overall, we have four equations and two free parameters. Hence, two factor exposures can arbitrary be chosen while the other two follow endogenously.

Corollary 1 treats the determination of the indirect utility function for the cases of full, partial, and no access to the derivatives market by applying Propositions 1 and 2. It takes into account the implications for the factor exposures which follow from (19), (20), and (21). Recall that it holds $\phi_{k}=\frac{h_{k}^{\mathcal{Q}} \lambda^{\mathcal{Q}}}{h_{k}^{\mathcal{P}} \lambda^{\mathcal{P}}}$.

Corollary 1. The indirect utility function is given by

$$
\mathcal{J}\left(\Pi, V_{1}, V_{2}\right)=\frac{\Pi^{1-\gamma}}{1-\gamma} e^{\gamma \bar{H}(\tau)},
$$

with

$$
\begin{aligned}
\bar{H}(\tau) & =\frac{1-\gamma}{\gamma} \tau\left[R+\sum_{j=1}^{J} \theta_{j}^{(W) *} \eta_{j}-\bar{\lambda}^{\mathcal{Q}} \sum_{k=1}^{K} \theta_{k}^{(N) *} h_{k}^{\mathcal{Q}}\right] \\
& -\frac{1}{2}(1-\gamma) \tau\left(\theta_{k}^{(W) *}\right)+\tau \bar{\lambda}^{\mathcal{P}} \sum_{k=1}^{K} \frac{\left(1+\theta_{k}^{(N) *}\right)-1}{\gamma} h_{k}^{\mathcal{P}} \\
\theta_{j}^{(W) *} & =\frac{\eta_{j}}{\gamma} \\
\theta_{k}^{(N) *} & =\left(\frac{1}{\phi_{k}}\right)^{\frac{1}{\gamma}}-1 .
\end{aligned}
$$


If there are no options on the second stock then equations (22) and (23) apply with

$$
\theta_{2}^{(W) *}=\frac{a_{2,2}}{\mu_{2}} \theta_{2}^{(N) *}
$$

and $\theta_{2}^{(N) *}$ satisfies

$$
\bar{\lambda}^{\mathcal{P}} h_{2}^{\mathcal{P}}\left(1+\theta_{2}^{(N) *}\right)^{-\gamma}=\frac{a_{2,2}}{\mu_{2}}\left[\theta_{2}^{(W) *} \gamma-\eta_{2}\right]+\bar{\lambda}^{\mathcal{Q}} h_{2}^{\mathcal{Q}}
$$

If there is no access to derivatives (22) and (23) hold with

$$
\begin{aligned}
\theta_{1}^{(W) *} & =\frac{a_{1,1}}{\mu_{1}} \theta_{1}^{(N) *}+\left(\frac{a_{2,1}}{\mu_{2}}-\frac{a_{1,1}}{\mu_{1}}\right) \theta_{2}^{(N) *} \\
\theta_{2}^{(W) *} & =\frac{a_{2,2}}{\mu_{2}} \theta_{2}^{(N) *}
\end{aligned}
$$

and $\theta_{1}^{(N) *}$ and $\theta_{2}^{(N) *}$ satisfy

$$
\begin{aligned}
\bar{\lambda}^{\mathcal{P}} h_{1}^{\mathcal{P}}\left(1+\theta_{1}^{(N) *}\right)^{-\gamma} & =\frac{a_{1,1}}{\mu_{1}}\left[\theta_{1}^{(W) *} \gamma-\eta_{1}\right]+\bar{\lambda}^{\mathcal{Q}} h_{1}^{\mathcal{Q}} \\
\bar{\lambda}^{\mathcal{P}} h_{2}^{\mathcal{P}}\left(1+\theta_{2}^{(N) *}\right)^{-\gamma} & =\left(\frac{a_{2,1}}{\mu_{2}}-\frac{a_{1,1}}{\mu_{1}}\right)\left[\theta_{1}^{(W) *} \gamma-\eta_{1}\right] \\
& +\frac{a_{2,2}}{\mu_{2}}\left[\theta_{2}^{(W) *} \gamma-\eta_{2}\right]+\bar{\lambda}^{\mathcal{Q}} h_{2}^{\mathcal{Q}} .
\end{aligned}
$$

For the model discussed in this section analytical pricing formulas for plain vanilla call and put options are well known and given in the appendix. We consider the following parameter specification. First, we assume that the correlation between stock prices as well as both stock price volatilities excluding jump risk are 0.7 and 0.3 respectively. Thus, we select $a_{1,1}=0.3, a_{2,1}=0.7 \times 0.3=0.21$ and $a_{2,2}=\sqrt{1-0.7^{2}} \times 0.3 \approx 0.21$. The conditional probability of a common jump is 0.8 under both the empirical and the risk neutral measure, i.e. $h_{1}^{\mathcal{P}}=h_{1}^{\mathcal{Q}}=0.8$. The jump probabilities are $\lambda^{\mathcal{P}}=0.1$ and $\lambda^{\mathcal{Q}}=0.5$ which implies $\phi_{1}=\phi_{2}=5$. Finally, we set $\mu_{1}=\mu_{2}=-0.2$ and $\eta_{1}=\eta_{2}=0.3$.

The results are summarized in figures 1 to 6 . As indicated by figure 1 the relative importance of including an additional derivative on stock 2 depends to a large degree on the conditional probability of a joint jump: The higher $h_{1}^{\mathcal{P}}=h_{1}^{\mathcal{Q}}$ the less 
necessary the introduction of an additional derivative security becomes. For the base

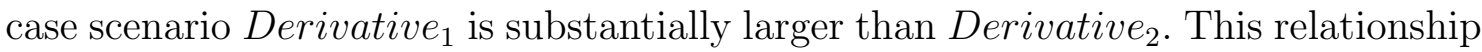
holds regardless of the choice of the risk aversion coefficient $\gamma$ as can be inferred from figure 2. Furthermore, diffusion correlation is analyzed in figures 3 and 4 where we distinguish two cases: In figure 3, we set the probability of a common jump according to the base case scenario, i.e. $h_{1}^{\mathcal{P}}=h_{1}^{\mathcal{Q}}=0.8$. In figure 4 this probability is 0.5. Interestingly, the "break even" correlation for which Derivative $_{1}>$ Derivative $_{2}$ is much larger in figure 4 . Nevertheless, the shape of these improvement curves is the same. This highlights the fact that both common jump probability and diffusion correlation have important implications on potential portfolio improvements through the introduction of derivatives. Finally, figures 5 and 6 show the relationships between Derivative D $_{1}$ and Derivative 2 and the jump width $\mu_{2}$ as well as the diffusion volatility of stock 2. Again, we find that the importance of additional derivatives depends critically on the choice of these parameters.

\section{Conclusion}

In this paper we address portfolio choice with derivatives in a generalized framework. We consider a model with several stocks and state variables which might be subject to stochastic jump and volatility risk. In the context of portfolio optimization we end up with a system of ordinary differential equations which can be solved either analytically or by standard numerical procedures. Furthermore, we distinguish between full and partial access to the derivatives market in order to measure the contribution of the introduction of individual derivative securities.

In the presence of jumps, exposures with respect to jump and diffusion factors cannot be chosen independently. This has an impact on the necessity of introducing new derivatives. Our numerical application deals with the jump diffusion model demonstrates that the importance of additional derivatives mainly depends on the conditional probability of a common jump of stock prices. The larger this conditional probability the less additional value is created by introducing new derivative securities. Other relevant parameters are diffusion correlation, jump widths, and diffusion volatilities.

Our results might be especially relevant for futures and options exchanges considering the introduction of new contracts for trade. We analyze under which circumstances a new product might become successful in the sense that liquid trading is 
established. Our focus was on portfolio optimization within a continuous time model in a frictionless market. Certainly, these requirements are not directly applicable to most market participants. We argue that products should not be introduced if potential portfolio improvement is small according to our model. Although this gives us some intuition about how exchanges should analyze product innovations a more explicit consideration of market frictions such as transaction costs would shed additional light into the matter. Furthermore, in our model we make the simplifying assumption that there are no limits on single portfolio positions. However, especially the use of derivatives is highly regulated in reality leading to limits on the amount to be spend on derivatives for many funds. Therefore, another extension would be to take into account these limits on the position in derivatives more explicitly. Finally, an interesting new research would also be an empirical applications of our results. 


\section{References}

Arditti, F. and John, K. (1980), 'Spanning the state space with options', Journal of Financial and Quantitative Analysis 15, 1-9.

Bakshi, G., Cao, C. and Chen, Z. (1997), 'Empirical performance of alternative option pricing models', Journal of Finance 52(5), 2003-2049.

Bakshi, G. and Madan, D. (2000), 'Spanning and derivative-security valuation', Journal of Financial Economics 55, 205-238.

Björk, T., Kabanov, Y. and Runggaldier, W. (1997), 'Bond market structure in the presence of marked point processes', Mathematical Finance 7(2), 211-223.

Branger, N., Schlag, C. and Schneider, E. (2005), Optimal portfolios when volatility can jump. Working Paper, University of Frankfurt.

Breeden, D. T. and Litzenberger, R. (1978), 'Prices of state-contingent claims implicit in options prices', Journal of Business 51, 621-651.

Campbell, J. Y. (1993), 'Intertemporal asset pricing without consumption data', American Economic Review 83, 487-512.

Campbell, J. Y. and Viceira, L. M. (1999), 'Consumption and portfilio decisions when expected returns are time varying', Quarterly Journal of Economics 114, 433-495.

Carr, P., Jin, X. and Madan, D. (2001), 'Optimal investment in derivative securities', Finance and Stochastics 5, 33-59.

Chacko, G. and Viceira, L. M. (2005), 'Dynamic consumption and portfolio choice with stochastic volatility in incomplete markets', Review of Financial Studies 18(4), 1369-1402.

Cox, J. C. and Huang, C.-f. (1989), 'Optimal consumption and portfolio choice when assets follow a diffusion process', Journal of Economic Theory 49, 33-83.

Daglesh, T. (2002), The economic significance of jump diffusions: Portfolio allocations. Working Paper, University of Toronto.

Duffie, D. and Kan, R. (1996), 'A yield factor model of interest rates', Mathematical Finance 6(4), 379-406. 
Green, R. and Jarrow, R. (1987), 'Spanning and completeness in markets with contingent claims', Journal of Economic Theory 41, 202-210.

Haugh, M. and Lo, A. (2001), 'Asset allocation and derivatives', Quantitative Finance 1, 45-72.

Heston, S. (1993), 'A closed-form solution for options with stochastic volatility with applications to bond and currency options', Review of Financial Studies 6(2), 327343.

Liu, J. (2005), Portfolio selection in stochastic environments. Working Paper, USLA.

Liu, J., Longstaff, F. A. and Pan, J. (2003), 'Dynamic asset allocation with event risk', Journal of Finance 53(1), 231-259.

Liu, J. and Pan, J. (2003), 'Dynamic derivative strategies', Journal of Financial Economics 69.

Merton, R. C. (1969), 'Lifetime portfolio selection under uncertainty: the continuous time case', Review of Economics and Statistics 51, 247-257.

Merton, R. C. (1971), 'Optimum consumption and portfolio rules in a continuoustime model', Journal of Economic Theory 3(4), 373-413.

Merton, R. C. (1973), 'Theory of rational option pricing', Bell Journal of Economics and Management Science 4(1), 141-183.

Merton, R. C. (1976), 'Option pricing when the underlying stock returns are discontinuous', Journal of Financial Economics 3(1-2), 125-144.

Munk, C. and Sorensen, C. (2004), 'Optimal consumption and investment strategies with stochastic interest rates', Journal of Banking and Finance 28, 1987-2013.

Ross, S. A. (1976), 'The arbitrage theory of capital asset pricing', Journal of Economic Theory 13, 341-360.

Rudolf, M. and Ziemba, W. T. (2004), 'Intertemporal surplus management', Journal of Economic Dynamics 6 Control 28, 975-990.

Wu, L. (2003), 'Jumps and dynamic asset allocation', Journal of Quantitative Finance and Accounting 20, 207-243. 


\section{Appendix}

\subsection{Proof of Lemma 1}

Consider the price $O_{u}$ of the $u$ th derivative. According to the pricing kernel specified in (2) it follows the stochastic process

$$
\begin{aligned}
& d O_{u}= \\
& \quad\left[R O_{u}+\sum_{k=0}^{K} \Delta_{k} O_{u}\left(\nu^{\mathcal{P}} h_{k}^{\mathcal{P}}-\nu^{\mathcal{Q}} h_{k}^{\mathcal{Q}}\right)\right] d t \\
& \quad+\sum_{i=1}^{I} \frac{\partial O_{u}}{\partial S_{i}} \sum_{j=1}^{J} S_{i} a_{i, j} \sqrt{V_{j}}\left(d W_{j}^{\mathcal{P}}+\eta_{j} \sqrt{V_{j}} d t\right) \\
& \quad+\sum_{l=1}^{L} \frac{\partial O_{u}}{\partial X_{l}} \sum_{j=1}^{J+L} b_{l, j} \sqrt{V_{j}}\left(d W_{j}^{\mathcal{P}}+\eta_{j} \sqrt{V_{j}} d t\right) \\
& +\sum_{k=1}^{K} \Delta_{k} O_{u}\left(d N_{k}-\nu^{\mathcal{P}} h_{k}^{\mathcal{P}} d t\right),
\end{aligned}
$$

Re-arranging yields:

$$
\begin{aligned}
& \frac{d O_{u}}{O_{u}}= \\
& R d t+\frac{1}{O_{u}}\left[\sum_{j=1}^{J} \sum_{i=1}^{I} \frac{\partial O_{u}}{\partial S_{i}} S_{i} a_{i, j}\left(\sqrt{V_{j}} d W_{j}^{\mathcal{P}}+\eta_{j} V_{j} d t\right)\right] \\
& +\frac{1}{O_{u}}\left[\sum_{l=1}^{L} \sum_{j=1}^{J+L} \frac{\partial O_{u}}{\partial X_{l}} b_{l, j}\left(\sqrt{V_{j}} d W_{j}^{\mathcal{P}}+\eta_{j} V_{j} d t\right)\right] \\
& +\frac{1}{O_{u}} \sum_{k=1}^{K} \Delta_{k} O_{u} d Z_{k} .
\end{aligned}
$$

In order to be self-financing no cash in- or outflows are allowed for the wealth process. Given the stochastic processes for the option (29) and the stock prices (1) and taking into account that portfolio weights must add up to one the wealth process 
turns out to be

$$
\begin{aligned}
& \frac{d \Pi}{\Pi}= \\
& R d t+\sum_{j=1}^{J}\left[\sum_{i=1}^{I} \omega_{i} a_{i, j}+\sum_{u=1}^{U} \psi_{u} \frac{1}{O_{u}}\left(\sum_{i=1}^{I} \frac{\partial O_{u}}{\partial S_{i}} a_{i, j} S_{i}+\sum_{l=1}^{L} \frac{\partial O_{u}}{\partial X_{l}} b_{l, j}\right)\right] \\
& \times\left(\sqrt{V_{j}} d W_{j}^{\mathcal{P}}+\eta_{j} V_{j} d t\right) \\
& +\sum_{j=J+1}^{J+L} \sum_{u=1}^{U} \psi_{u} \sum_{l=1}^{L} \frac{1}{O_{u}} \frac{\partial O_{u}}{\partial X_{l}} b_{l, j}\left(\sqrt{V_{j}} d W_{j}^{\mathcal{P}}+\eta_{j} V_{j} d t\right) \\
& +\sum_{k=1}^{K}\left[\sum_{i=1}^{I} \omega_{i} \mu_{i, k}+\sum_{u=1}^{U} \psi_{u} \frac{1}{O_{u}} \Delta_{k} O_{u}\right] d Z_{k},
\end{aligned}
$$

Inserting the definitions for $\theta^{(\cdot)}$ yields lemma 1 .

\subsection{Proof of Proposition 1}

The portfolio choice problem is solved by applying the principle of stochastic control.

The stochastic wealth process is given by lemma 1. The Hamilton/Jacobi/Bellman (HJB) equation follows as

$$
\begin{aligned}
& \max _{\theta^{(\cdot)}}\left\{f_{\mathrm{HJB}}\right\}=0 \\
& f_{\mathrm{HJB}}=\frac{\partial \mathcal{J}}{\partial t} \\
& +\Pi \frac{\partial \mathcal{J}}{\partial \Pi}\left[R+\sum_{j=1}^{J+L} V_{j} \theta_{j}^{(W)} \eta_{j}-\nu^{\mathcal{Q}} \sum_{k=1}^{K} \theta_{k}^{(N)} h_{k}^{\mathcal{Q}}\right]+\frac{1}{2} \frac{\partial^{2} \mathcal{J}}{\partial \Pi^{2}} \Pi^{2} \sum_{j=1}^{J+L} V_{j}\left(\theta_{j}^{(W)}\right)^{2} \\
& +\sum_{l=1}^{L}\left[\frac{\partial \mathcal{J}}{\partial X_{l}} m_{l}^{\mathcal{P}}+\frac{1}{2} \sum_{u=1}^{L} \sum_{j=1}^{J+L} \frac{\partial^{2} \mathcal{J}}{\partial X_{l} \partial X_{u}} b_{l, j} b_{u, j} V_{j}+\Pi \frac{\partial^{2} \mathcal{J}}{\partial \Pi \partial X_{l}} \sum_{j=1}^{J+L} b_{l, j} V_{j} \theta_{j}^{(W)}\right] \\
& +\nu^{\mathcal{P}} \sum_{k=1}^{K} \Delta_{k} \mathcal{J} h_{k}^{\mathcal{P}},
\end{aligned}
$$


where $\Delta_{k} \mathcal{J}=\mathcal{J}\left(\left(1+\theta_{k}^{(N)}\right) \Pi, X_{1}\left(1+\mu_{J+1, k}\right), \ldots, X_{1}\left(1+\mu_{J+L, k}\right)\right)-\mathcal{J}(\Pi)$.

We conjecture the functional form of the indirect utility function as given in (6). Using this, the derivatives of $\mathcal{J}$ as well as $\Delta_{k} \mathcal{J}$ can be computed and the optimization problem (31) reduces to

$$
\begin{aligned}
& \max _{\theta^{(\cdot)}}\left\{f_{\mathrm{HJB}}\right\}=0 \\
& \frac{f_{\mathrm{HJB}}}{\gamma \mathcal{J}}=\frac{\partial \bar{H}}{\partial t}+\sum_{l=1}^{L} \frac{\partial H_{l}}{\partial t} X_{l} \\
& +\frac{1-\gamma}{\gamma}\left[R+\sum_{j=1}^{J+L} V_{j} \theta_{j}^{(W)} \eta_{j}-\nu^{\mathcal{Q}} \sum_{k=1}^{K} \theta_{k}^{(N)} h_{k}^{\mathcal{Q}}\right] \\
& -\frac{1}{2}(1-\gamma) \sum_{j=1}^{J+L} V_{j}\left(\theta_{j}^{(W)}\right)^{2} \\
& +\sum_{l=1}^{L}\left[H_{l} m_{l}^{\mathcal{P}}+\frac{1}{2} \gamma \sum_{u=1}^{L} \sum_{j=1}^{J+L} b_{l, j} b_{u, j} H_{l} H_{u} V_{j}+(1-\gamma) H_{l} \sum_{j=1}^{J+L} b_{l, j} V_{j} \theta_{j}^{(W)}\right] \\
& +\frac{\nu^{\mathcal{P}}}{\gamma} \sum_{k=1}^{K}\left[\left(1+\theta_{k}^{(N)}\right)^{1-\gamma} e^{\left.\gamma \sum_{u=1}^{L} H_{u} \mu_{I+u, k}-1\right] h_{k}^{\mathcal{P}} .}\right.
\end{aligned}
$$

In order to maximize $f_{\mathrm{HJB}}$ the first order conditions must be applied. The optimal portfolio weights stated in equation (8) in proposition 1 follow. Inserting the optimal exposures (8) into (32) and using the definitions of $V_{j}, R$, and $\nu^{(\cdot)}$ in (1) finally leads to the system of ordinary differential equations (7) which completes the proof.

\subsection{Proof of Proposition 2}

The problem can be solved by applying the principle of stochastic control. The HJB equation is a special case of (31) with $L=0, V_{j}=1$, and the indirect utility function 
being independent of any state variables other then wealth $\Pi$

$$
\begin{aligned}
& \max _{\theta^{(\cdot)}}\left\{f_{\mathrm{HJB}}\right\}=0 \\
& f_{\mathrm{HJB}}=\frac{\partial \mathcal{J}}{\partial t} \\
& +\Pi \frac{\partial \mathcal{J}}{\partial \Pi}\left[R+\sum_{j=1}^{J} \theta_{j}^{(W)} \eta_{j}-\bar{\lambda}^{\mathcal{Q}} \sum_{k=1}^{K} \theta_{k}^{(N)} h_{k}^{\mathcal{Q}}\right] \\
& +\frac{1}{2} \frac{\partial^{2} \mathcal{J}}{\partial \Pi^{2}} \Pi^{2} \sum_{j=1}^{J}\left(\theta_{j}^{(W)}\right)^{2}+\bar{\lambda}^{\mathcal{P}} \sum_{k=1}^{K} \Delta_{k} \mathcal{J} h_{k}^{\mathcal{P}} .
\end{aligned}
$$

Conjecturing the indirect utility function according to (11) and applying the first order conditions leads to the optimal factor exposures given by (13) and (14). Inserting the optimal exposures into (33) leads to (12).

\subsection{Option Pricing Section 4}

Consider the stock price process (18). Let $Y_{i}=\ln S_{i}$. Then under the risk neutral measure we have

$$
\begin{aligned}
d Y_{i} & =\left\{r-\frac{1}{2}\left[a_{i, 1}^{2}+a_{i, 2}^{2}\right]-\widetilde{\lambda}_{i}^{\mathcal{Q}} \mu_{i}\right\} d t+a_{i, 1} d W_{1}^{\mathcal{Q}}+a_{i, 2} d W_{2}^{\mathcal{Q}} \\
& +\mu_{i} d \widetilde{N}_{i}
\end{aligned}
$$

where $\widetilde{N}_{1}$ and $\widetilde{N}_{2}$ are counting processes with intensities $\widetilde{\lambda}_{1}^{\mathcal{Q}}=\bar{\lambda}^{\mathcal{Q}} h_{1}$ and $\widetilde{\lambda}_{2}^{\mathcal{Q}}=\bar{\lambda}^{\mathcal{Q}}$ respectively. The price of a call option on stock $i$ with strike $H$ and expiry in $\tau$ can 
be calculated by applying the results of Merton (1976)

$$
\begin{aligned}
\operatorname{Call}_{i}(H, \tau) & =\sum_{n=0}^{\infty} \frac{e^{-\tilde{\lambda}^{\mathcal{Q}}\left(1+\mu_{i}\right) \tau}\left(\widetilde{\lambda}^{\mathcal{Q}}\left(1+\mu_{i}\right) \tau\right)^{n}}{n !} c_{n}(H, \tau) \\
c_{n}(H, \tau) & =S_{i} \Phi\left[d_{1}(n)\right]-H e^{-r_{n} \tau} \Phi\left[d_{2}(n)\right] \\
\sigma_{D} & =\sqrt{a_{i, 1}^{2}+a_{i, 2}^{2}} \\
r_{n} & =r-\widetilde{\lambda}_{i}^{\mathcal{Q}} \mu_{i}+\frac{n \ln \left(1+\mu_{i}\right)}{\tau} \\
d_{1}(n) & =\frac{\ln \frac{S_{i}}{H}+\left(\frac{1}{2} \sigma_{D}^{2}+r_{n}\right) \tau}{\sigma_{D} \sqrt{\tau}} \\
d_{2}(n) & =d_{1}(n)-\sigma_{D} \sqrt{\tau},
\end{aligned}
$$

where $\Phi[\cdot]$ is the operator for the cumulative standard normal probability distribution. 
Figure 1: Probability Common Jump

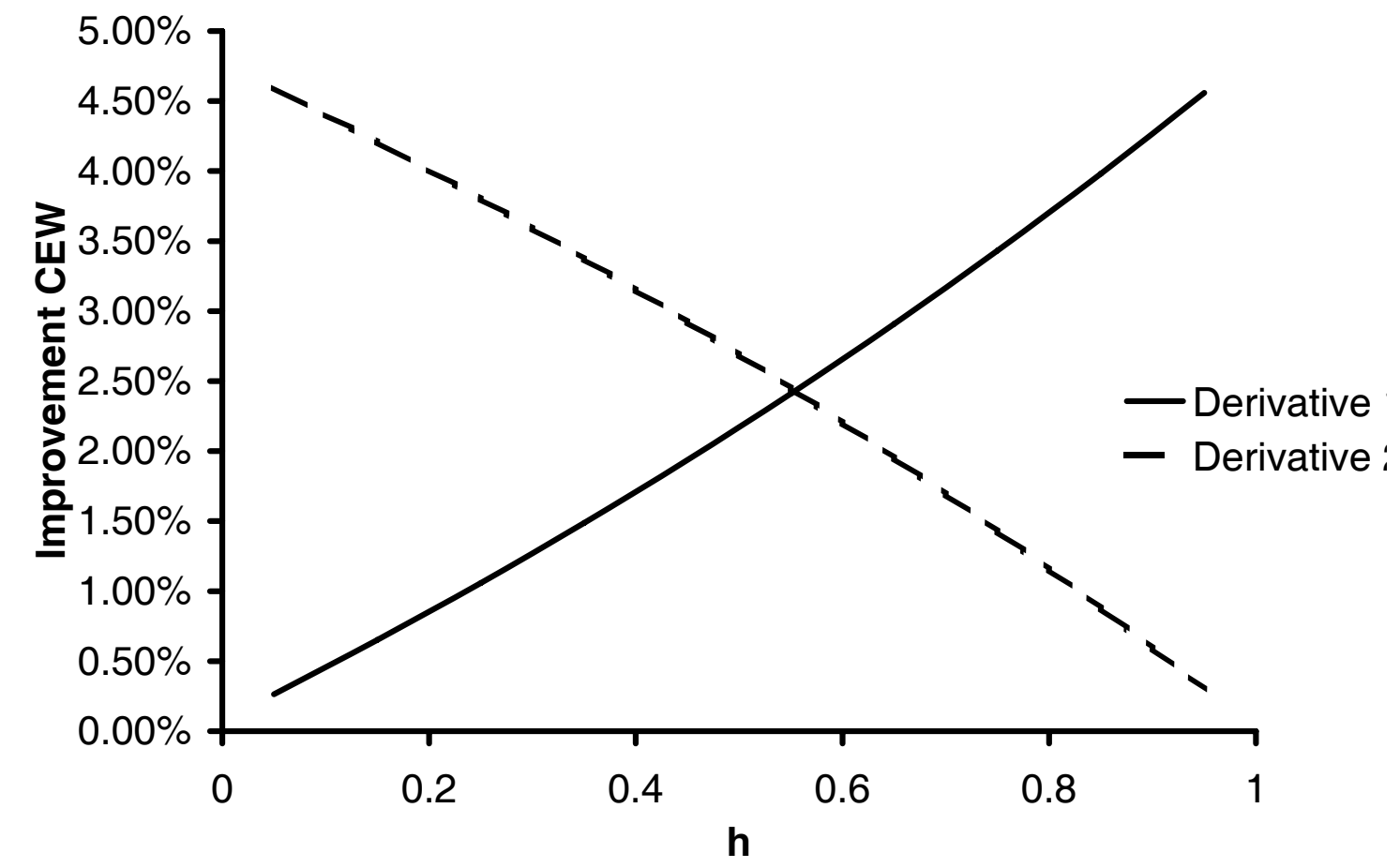

Derivative 1 and Derivative 2 show the portfolio improvements from including derivatives according to (17). The base case is defined as $r=5 \%, \tau=1$ year, $\gamma=4, \eta_{1}=\eta_{2}=0.3, \lambda^{\mathcal{P}}=0.1, \lambda^{\mathcal{Q}}=0.5$, $h_{1}^{\mathcal{P}}=h_{1}^{\mathcal{Q}}=0.8, h_{2}^{\mathcal{P}}=h_{2}^{\mathcal{Q}}=0.8$, and $\mu_{1}=\mu_{2}=-0.2$. The diffusion volatilities and correlation are 0.3 and 0.7 respectively. 
Figure 2: Risk Aversion

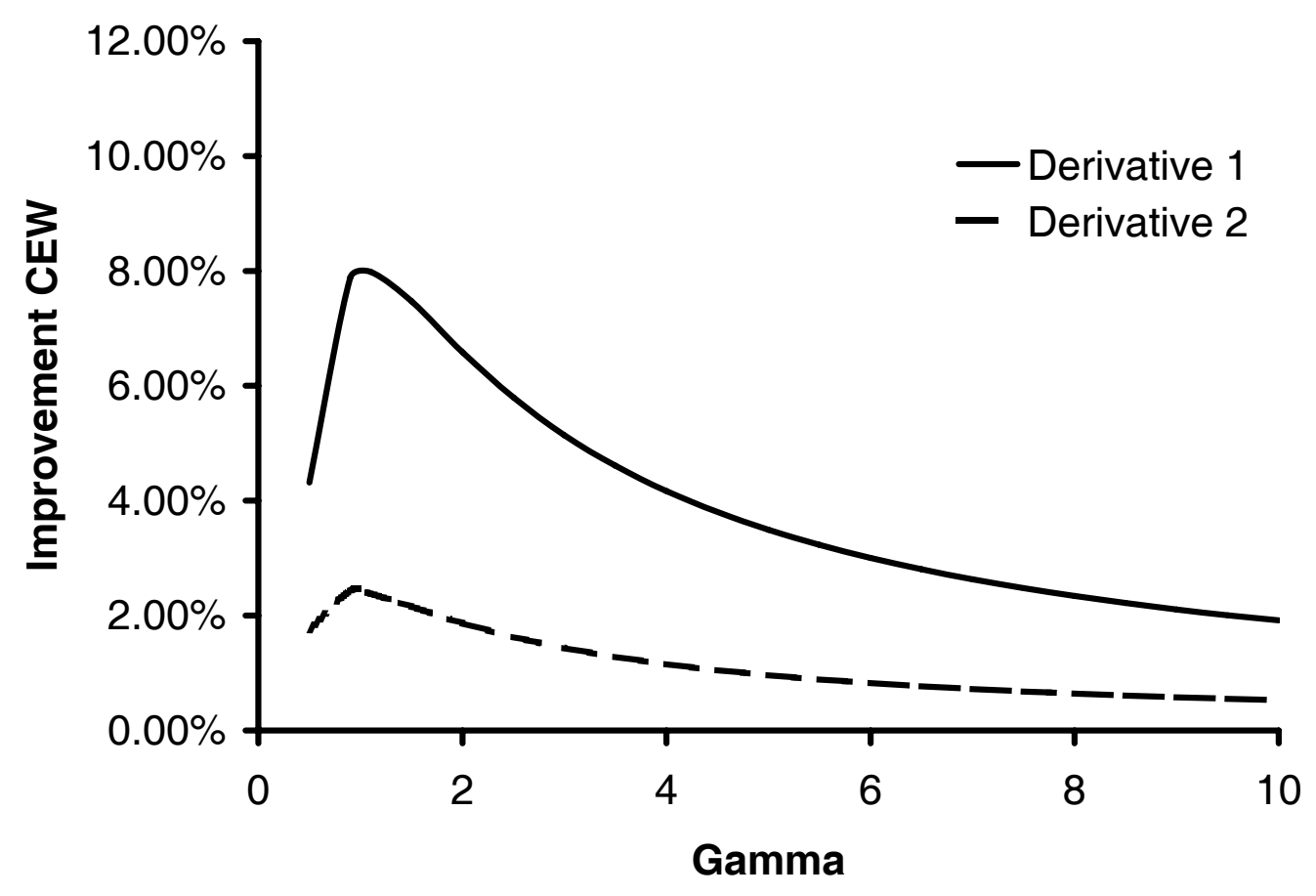

Derivative 1 and Derivative 2 show the portfolio improvements from including derivatives according to (17). The base case is defined as $r=5 \%, \tau=1$ year, $\gamma=4, \eta_{1}=\eta_{2}=0.3, \lambda^{\mathcal{P}}=0.1, \lambda^{\mathcal{Q}}=0.5$, $h_{1}^{\mathcal{P}}=h_{1}^{\mathcal{Q}}=0.8, h_{2}^{\mathcal{P}}=h_{2}^{\mathcal{Q}}=0.8$, and $\mu_{1}=\mu_{2}=-0.2$. The diffusion volatilities and correlation are 0.3 and 0.7 respectively. 
Figure 3: Correlation Base Case

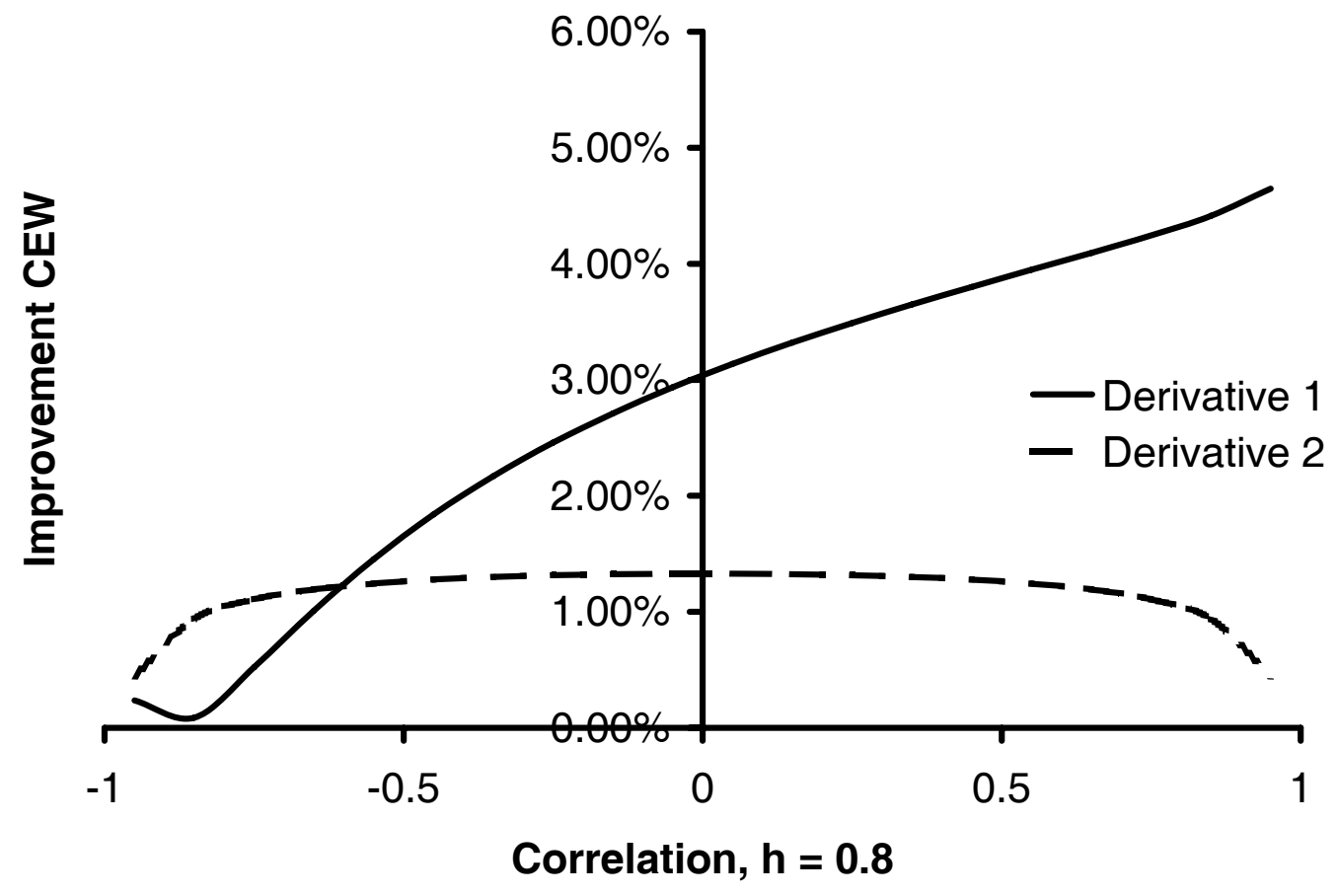

Derivative 1 and Derivative 2 show the portfolio improvements from including derivatives according to (17). The base case is defined as $r=5 \%, \tau=1$ year, $\gamma=4, \eta_{1}=\eta_{2}=0.3, \lambda^{\mathcal{P}}=0.1, \lambda^{\mathcal{Q}}=0.5$, $h_{1}^{\mathcal{P}}=h_{1}^{\mathcal{Q}}=0.8, h_{2}^{\mathcal{P}}=h_{2}^{\mathcal{Q}}=0.8$, and $\mu_{1}=\mu_{2}=-0.2$. The diffusion volatilities and correlation are 0.3 and 0.7 respectively. 
Figure 4: Correlation, $h_{1}^{\mathcal{P}}=h_{1}^{\mathcal{Q}}=0.5$

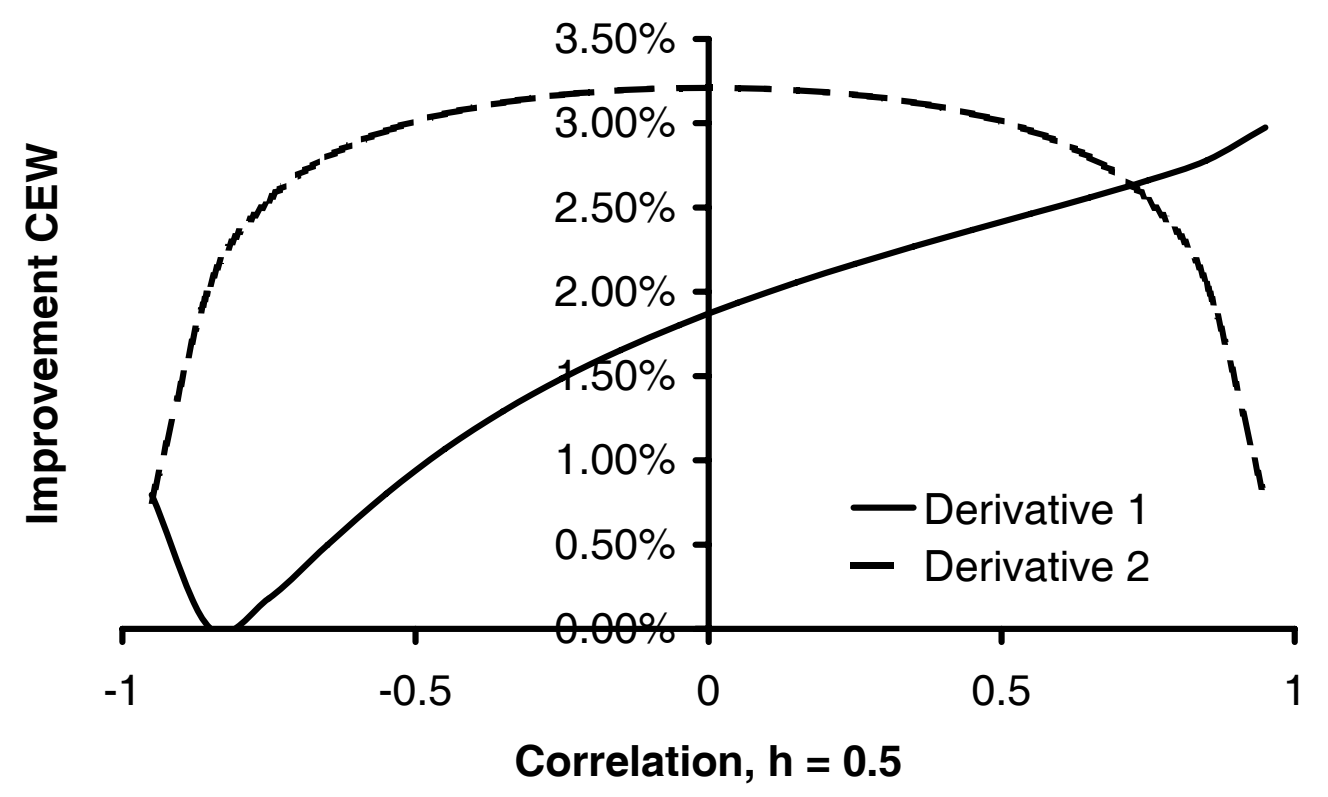

Derivative 1 and Derivative 2 show the portfolio improvements from including derivatives according to (17). The base case is defined as $r=5 \%, \tau=1$ year, $\gamma=4, \eta_{1}=\eta_{2}=0.3, \lambda^{\mathcal{P}}=0.1, \lambda^{\mathcal{Q}}=0.5$, $h_{1}^{\mathcal{P}}=h_{1}^{\mathcal{Q}}=0.5, h_{2}^{\mathcal{P}}=h_{2}^{\mathcal{Q}}=0.5$, and $\mu_{1}=\mu_{2}=-0.2$. The diffusion volatilities and correlation are 0.3 and 0.7 respectively. 
Figure 5: Jump Amplitude $\mu_{2}$

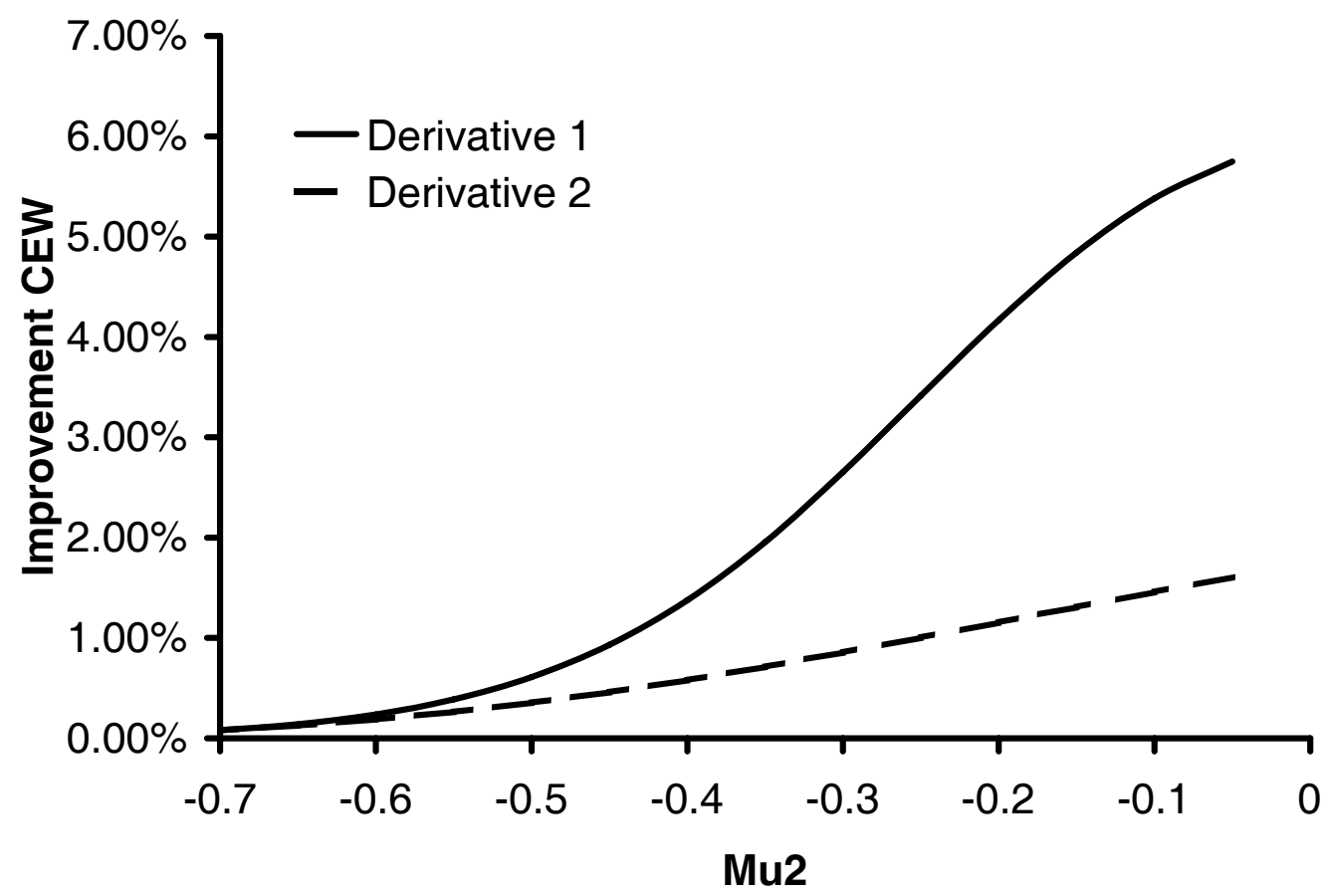

Derivative 1 and Derivative 2 show the portfolio improvements from including derivatives according to (17). The base case is defined as $r=5 \%, \tau=1$ year, $\gamma=4, \eta_{1}=\eta_{2}=0.3, \lambda^{\mathcal{P}}=0.1, \lambda^{\mathcal{Q}}=0.5$, $h_{1}^{\mathcal{P}}=h_{1}^{\mathcal{Q}}=0.8, h_{2}^{\mathcal{P}}=h_{2}^{\mathcal{Q}}=0.8$, and $\mu_{1}=\mu_{2}=-0.2$. The diffusion volatilities and correlation are 0.3 and 0.7 respectively. 
Figure 6: Diffusin Volatility Stock 2

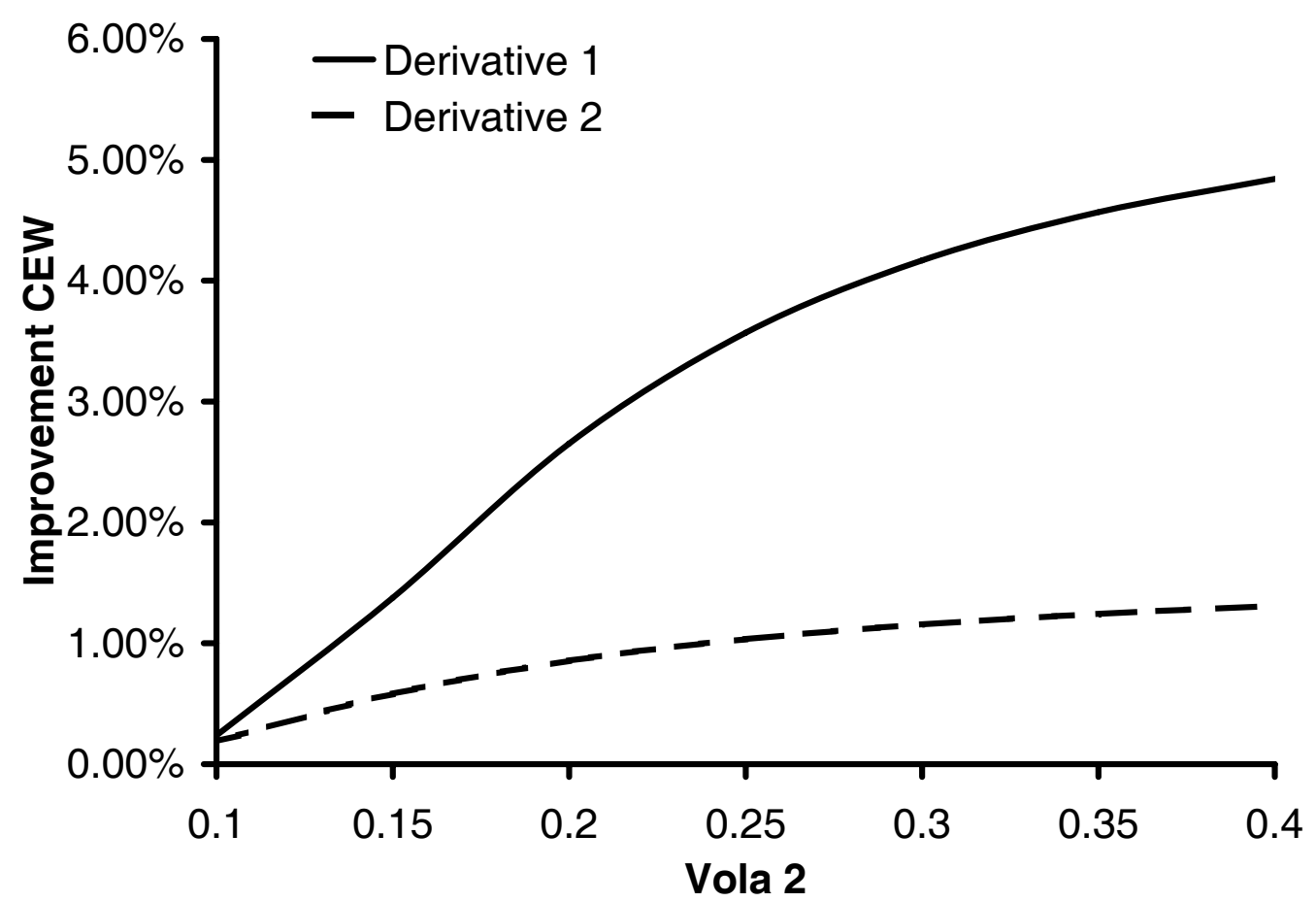

Derivative 1 and Derivative 2 show the portfolio improvements from including derivatives according to (17). The base case is defined as $r=5 \%, \tau=1$ year, $\gamma=4, \eta_{1}=\eta_{2}=0.3, \lambda^{\mathcal{P}}=0.1, \lambda^{\mathcal{Q}}=0.5$, $h_{1}^{\mathcal{P}}=h_{1}^{\mathcal{Q}}=0.8, h_{2}^{\mathcal{P}}=h_{2}^{\mathcal{Q}}=0.8$, and $\mu_{1}=\mu_{2}=-0.2$. The diffusion volatilities and correlation are 0.3 and 0.7 respectively. 